\title{
Direct observation of reversible oxygen migration and phase transitions in ferroelectric $\mathrm{Hf}_{0.5} \mathrm{Zr}_{0.5} \mathrm{O}_{2}$ thin-film devices
}

Pavan Nukala $^{1}$, Majid Ahmadi ${ }^{2}$, Sytze de Graaf ${ }^{2}$, Bart J. Kooi ${ }^{2}$, Beatriz Noheda ${ }^{3}$, Henny Zandbergen ${ }^{4}$ and Yingfen $\mathrm{Wei}^{5}$

${ }^{1}$ IISc, United States, ${ }^{2}$ Zernike Institute for Advanced Materials, University of Groningen, United States, ${ }^{3}$ University of Groningen, Netherlands, ${ }^{4}$ Kavli Institute of Nanoscience, Faculty of Applied Sciences, Delft University of Technology, Delft, Netherlands, ${ }^{5}$ EPFL, Switzerland, Groningen, United States

Nanoscopic silicon-compatible ferroelectricity in hafnia-based thin-films (1) has triggered vast amount of fundamental research and brought back the interest in ferroelectric materials in microelectronics. This ferroelectricity exists even at film thicknesses as low as $1 \mathrm{~nm}$, and increases with decrease in size, a situation that was believed impossible and is opposite to the classical understanding of ferroelectricity. The spontaneous polarization observed in these films is ascribed generally to a metastable polar orthorhombic phase (Pca21, o-phase) (2). A higher energy rhombohedral phase (R3m/R3, r-phases) has been reported for epitaxial growth of $\mathrm{Hf}_{0.5} \mathrm{Zr}_{0.5} \mathrm{O}_{2}$ (HZO) on $\mathrm{La}_{0.67} \mathrm{Sr}_{0.33} \mathrm{MnO}_{3}$ (LSMO) buffered perovskite and on trigonal substrates (3), and on the former, remnant polarization $\left(\mathrm{P}_{r}\right)$ values as high as $35 \mu \mathrm{C} / \mathrm{cm}^{2}$ were measured (4). The pronounced effects of particle size reduction, surface effects, dopants, oxygen vacancies $\left(\ddot{\mathrm{V}}_{\mathrm{o}}\right)$, epitaxial strain and residual stresses at nanoscale have all been investigated as possible reasons to stabilize these otherwise metastable phases in thin films.

Vibrant research is being conducted on the mechanism of polarization switching. The dynamics of this switching are currently being explored by various authors through the lens of conventional ferroelectric switching. However, another feature of hafnia-based materials is that they also exhibit memristive hysteresis driven by oxygen conduction and associated redox reactions $(5,6)$. A few works have begun to explore the role of $\mathrm{V}_{\mathrm{o}}$ " migration and its possible role in ferroelectricity $(7,8)$. In other words, can extrinsic reasons such as $\mathrm{V}_{\mathrm{o}} \cdot$ migration be the cause of this non-conventional ferroelectricity.

In this regard, we resorted operando atomic scale electron microscopy investigations of the behavior of LSMO/HZO/LSMO capacitor stacks. These stacks were grown using pulsed laser deposition on conducting (Nb-doped) STO substrate under electric field. LSMO is a standard choice of bottom electrode in complex oxide devices and, thus, the findings reported here are relevant for understanding a wider class of devices. In situ biasing measurements were performed while simultaneously employing two scanning transmission electron microscopy (STEM) imaging modes: high-angle annular dark-field (HAADF) STEM and integrated differential phase contrast (iDPC) STEM. iDPC-STEM is currently the most robust atomic resolution imaging technique to measure simultaneously heavy and light elements. By directly imaging oxygen, we provide evidence of the reversible and hysteretic migration of $\ddot{\mathrm{V}}_{\mathrm{o}}$ from the bottom to the top electrode through the HZO layer. Associated with such migration, we show $\ddot{V}_{\mathrm{o}}$ induced phase transitions in LSMO (bottom electrode) and HZO layers. In combination with ex situ microscopy and transport measurements on real devices we clearly show that polarization switching, and oxygen voltammetry are intertwined.

[This work is currently published on https://arxiv.org/abs/2010.10849] 
$\mathbf{a}$

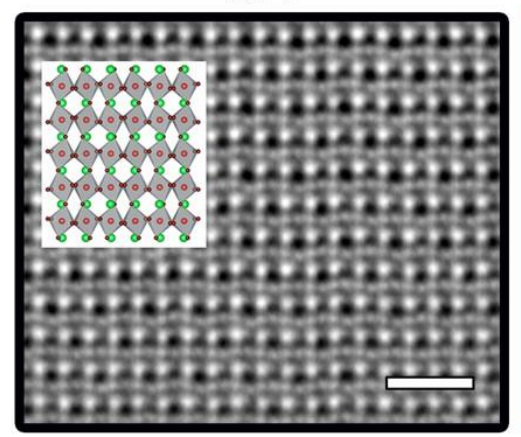

d
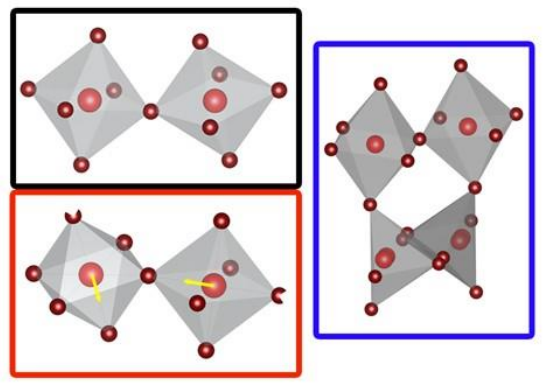

b

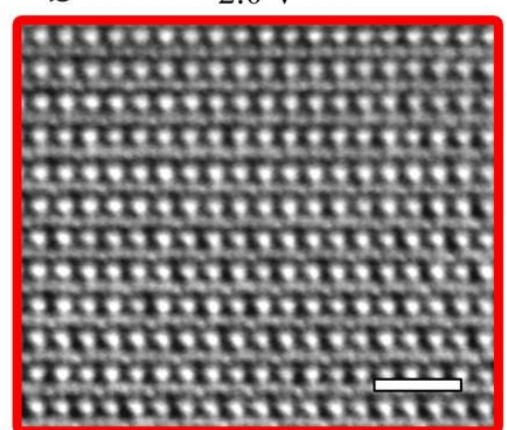

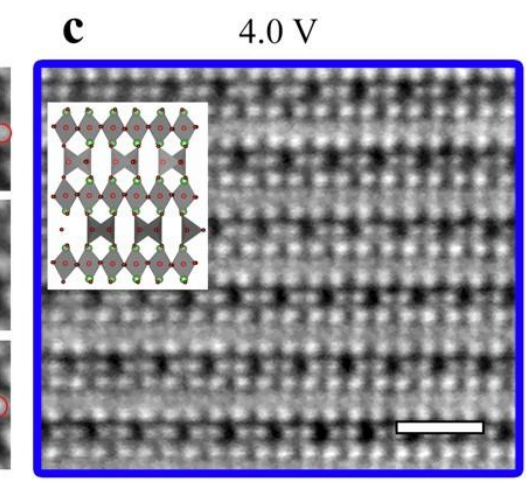

f
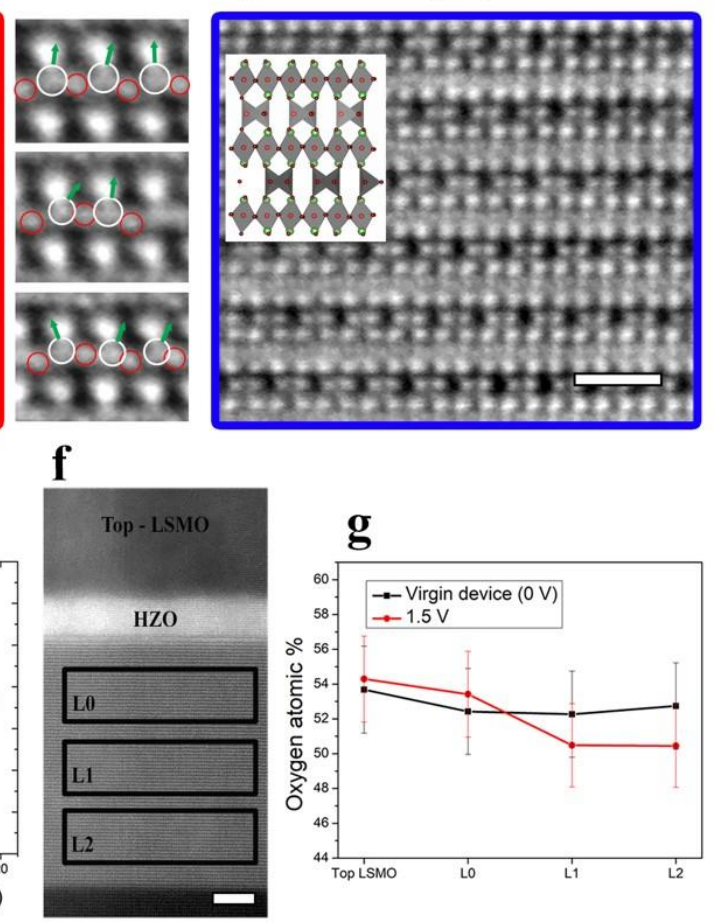

Figure 1. Figure 1: Deoxygenation of bottom electrode LSMO layer with increasing positive bias. (a) iDPC-STEM images of the entire $30 \mathrm{~nm}$ bottom LSMO region in the virgin state. Interfaces with $\mathrm{Nb}: \mathrm{STO}$ (bottom) and HZO (top) layers are marked. Zoomed up image of a select region (black square) is shown in the inset. Select oxygen positions are marked in brown circles. Two adjacent MnO6 octahedra are overlaid, illustrating the $\delta$-tilts. (b) iDPC-STEM images of at V=2 V. Region enclosed in red is zoomed in the inset. Select oxygen positions are marked in brown circles. Brownmillerite (BM) precursor phase is illustrated by marking the displacement of a few Mn atoms from the octahedral centers (red arrows). (c) iDPC-STEM at 4 V. BM LSMO (zone axis :a, schematic in inset) signified by alternating MnO4 tetrahedra and MnO6 octahedra. Scale bar, $1 \mathrm{~nm}$ for (a-c). (d) At 0V, BM phase is retained. Image shows two domains of BM LSMO, with zone axes, $c$ and a respectively, (inset) schematic of BM phase visualized with c axis as the zone. Scale bar, $2 \mathrm{~nm}$. (e) Plot of variation of c' (La-La distance) parameter from the STO interface in perovskite (green), BM-precursor (blue) and BM phases (red). (f) Overview image of LSMO/HZO/LSMO capacitor with regions marked where oxygen content was quantified from EDS (g) at $0 \mathrm{~V}$ and $1.5 \mathrm{~V}$. Scale bar, $5 \mathrm{~nm}$ in (f). 

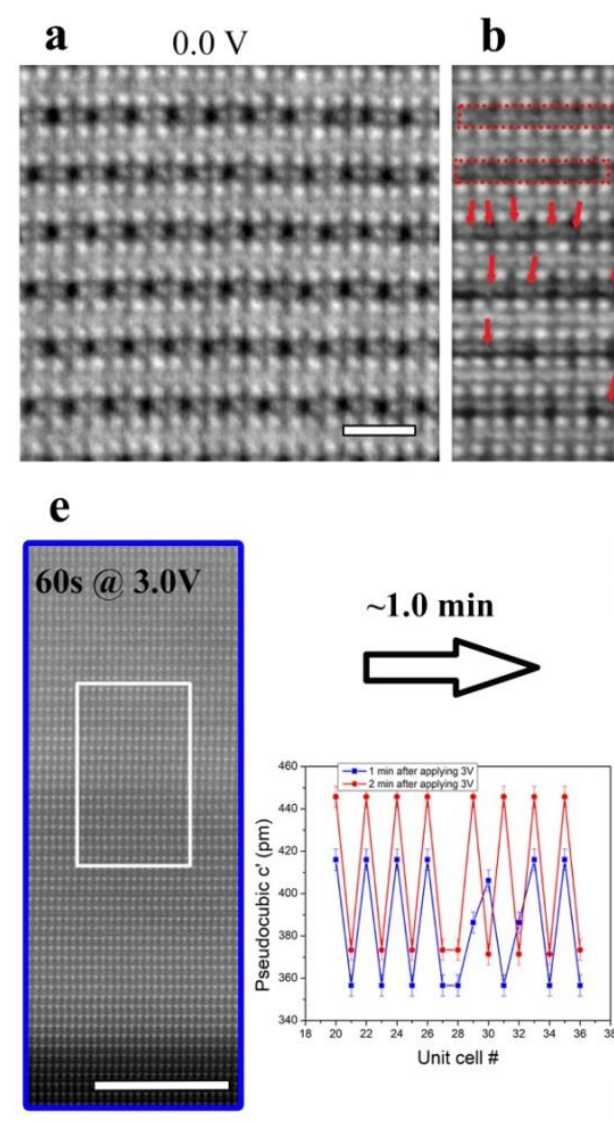
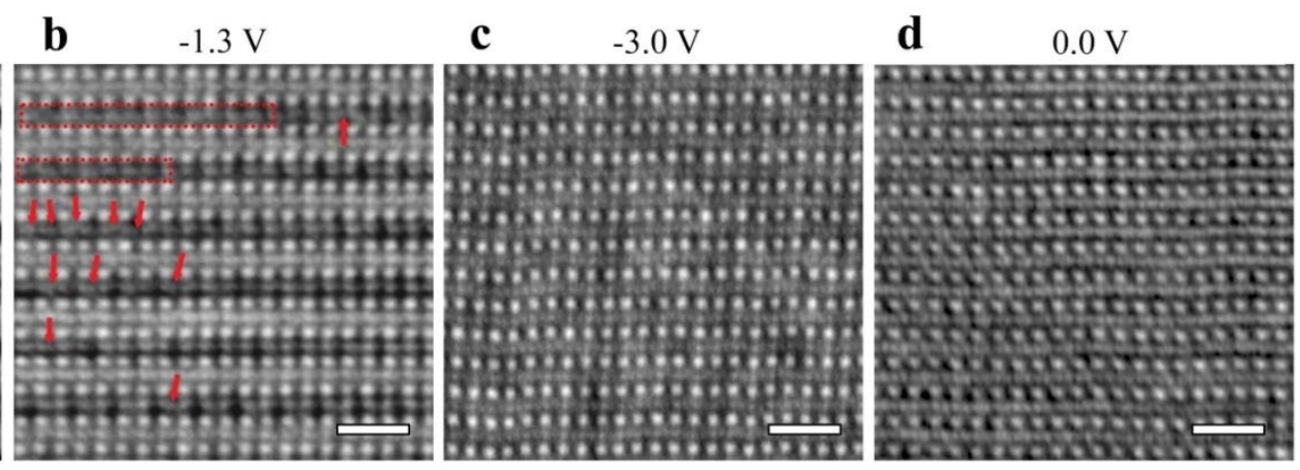

Figure 2. Figure 2: Reoxygenation of bottom electrode LSMO layer with increasing negative bias.a) iDPC-STEM images of one particular region at 0 and $-1.3 \mathrm{~V}$. At $-1.3 \mathrm{~V}$ oxygen columns start to appear (marked) in positions. Boundary with the substrate is marked. Scale bar, $2 \mathrm{~nm}$ (b) BM phase transforms to $\mathrm{BM}$ precursor phase at $-3 \mathrm{~V}$ and is retained so at $0 \mathrm{~V}(\mathrm{c}), \mathrm{BM}$ precusor phase also shows disorder in c' parameter variation (d). Scale bar, $1 \mathrm{~nm}$ in (b-c). (e) Dynamics of evolution followed through HAADFSTEM imaging within 120 seconds of ramping upto $3 \mathrm{~V}$. A BM-precursor phase is imaged at $60 \mathrm{sec}, \mathrm{BM}$ phase is imaged at $120 \mathrm{sec}$. (f) Upon changing the bias to $-3 \mathrm{~V}$, a BM phase recorded at 30 sec (with superstructure spots in the FFT), changes to perovskite phase by $90 \mathrm{sec}$ (disappearance of the superstructure spots). Scale bar, $2 \mathrm{~nm}$, (e-f). (g) The intermediate BM-precursor phase recorded at $60 \mathrm{sec}$, converts to perovskite-like phase in about $6 \mathrm{sec}$, giving an idea about the time scales of Vo.. migration.

\section{References}

1. T. S. Böscke, J. Müller, D. Bräuhaus, U. Schröder, U. Böttger, Ferroelectricity in hafnium oxide thin films. Appl. Phys. Lett. (2011), doi:10.1063/1.3634052.

2. U. Schröder, C. S. Hwang, H. Funakubo, Eds., Ferroelectricity in doped hafnium oxide (Woodhead Publishing 2019, ed. 1st).

3. P. Nukala, Y. Wei, V. de Haas, J. Antoja-Lleonart, Q. Guo, B. Noheda, Guidelines for the stabilization of a polar rhombohedral phase in epitaxial $\mathrm{Hf0.5Zr0.5O2}$ thin films. arXiv:2005.01809 (2020).

4. Y. Wei, P. Nukala, M. Salverda, S. Matzen, H. J. Zhao, J. Momand, A. S. Everhardt, G. Agnus, G. R. Blake, P. Lecoeur, B. J. Kooi, J. Íñiguez, B. Dkhil, B. Noheda, A rhombohedral ferroelectric phase in epitaxially strained Hf 0.5 Zr 0.5 O 2 thin films. Nat. Mater. (2018), doi:10.1038/s41563-018-0196-0. 
5. C. Li, Y. Yao, X. Shen, Y. Wang, J. Li, C. Gu, R. Yu, Q. Liu, M. Liu, Dynamic observation of oxygen vacancies in hafnia layer by in situ transmission electron microscopy. Nano Res. (2015), doi:10.1007/s12274-015-0857-0.

6. S. U. Sharath, S. Vogel, L. Molina-Luna, E. Hildebrandt, C. Wenger, J. Kurian, M. Duerrschnabel, T. Niermann, G. Niu, P. Calka, M. Lehmann, H. J. Kleebe, T. Schroeder, L. Alff, Control of Switching Modes and Conductance Quantization in Oxygen Engineered HfOx based Memristive Devices. Adv. Funct. Mater. (2017), doi:10.1002/adfm.201700432.

7. V. Mikheev, A. Chouprik, Y. Lebedinskii, S. Zarubin, Y. Matveyev, E. Kondratyuk, M. G. Kozodaev, A. M. Markeev, A. Zenkevich, D. Negrov, Ferroelectric Second-Order Memristor. ACS Appl. Mater. Interfaces (2019), doi:10.1021/acsami.9b08189.

8. M. D. Glinchuk, A. N. Morozovska, A. Lukowiak, W. Stręk, M. V. Silibin, D. V. Karpinsky, Y. Kim, S. V. Kalinin, Possible electrochemical origin of ferroelectricity in $\mathrm{HfO} 2$ thin films. J. Alloys Compd. (2020), doi:10.1016/j.jallcom.2019.153628. 